\title{
The Asymptotic Behavior of Solutions for 3D Globally Modified Bénard Problem with Delay
}

\author{
Xia Hou, Chaosheng Zhu* \\ School of Mathematics and Statistics, Southwest University, Chongqing, China \\ Email: 1518534126@qq.com, ${ }^{\star}$ zcs@swu.edu.cn
}

How to cite this paper: Hou, X. and Zhu, C.S. (2019) The Asymptotic Behavior of Solutions for 3D Globally Modified Bénard Problem with Delay. Open Access Library Journal, 6: e5163.

https://doi.org/10.4236/oalib.1105163

Received: January 2, 2019

Accepted: January 28, 2019

Published: January 31, 2019

Copyright $\odot 2019$ by author(s) and Open Access Library Inc.

This work is licensed under the Creative

Commons Attribution International

License (CC BY 4.0).

http://creativecommons.org/licenses/by/4.0/

\section{(c) (i) Open Access}

\begin{abstract}
In this paper, we mainly study the existence and uniqueness of solutions and the asymptotic behavior of solutions for three-dimensional globally modified Bénard systems with delays under local Lipschitz conditions.
\end{abstract}

\section{Subject Areas}

Fluid Mechanics, Partial Differential Equation

\section{Keywords}

Bénard System, Delay, Galerkin Approximation, Asymptotic Behavior

\section{Introduction}

Let $\Omega \in \mathbb{R}^{3}$ be an open and bounded set with regular boundary $\Gamma$. We consider the following problem for the Bénard system with homogeneous Dirichlet boundary conditions on $\Omega$ :

$$
\left\{\begin{array}{lc}
\frac{\partial u}{\partial t}-v \Delta u+(u \cdot \nabla) u+\xi \omega+\nabla p=f, & \text { in }(0,+\infty) \times \Omega, \\
\frac{\partial \omega}{\partial t}-\Delta \omega+(u \cdot \nabla) \omega=g, & \text { in }(0,+\infty) \times \Omega, \\
\nabla \cdot u=0, & \text { in }(0,+\infty) \times \Omega, \\
u=0, \omega=0, & \text { on }(0,+\infty) \times \partial \Omega, \\
u(x, 0)=u_{0}(x), \omega(x, 0)=\omega_{0}(x), & x \in \Omega .
\end{array}\right.
$$

Here $u(x, t), \omega(x, t)$ are respectively the velocity, temperature of the fluid, $p(x, t)$ is the pressure of the fluid, $v$ is the viscous coefficient of the fluid, $\xi \in \mathbb{R}^{3}$ is a constant vector, $u_{0}, \omega_{0}$ are the initial value. The external force terms $f: \mathbb{R} \times \Omega \rightarrow \mathbb{R}^{3}, g: \mathbb{R} \times \Omega \rightarrow \mathbb{R}$ are a given function.

As we all know, Bénard system consists of the Navier-Stokes equations 
coupled with a parabolic equation. This system is a well-known model in hydrodynamics. It describes the behavior of the velocity, the pressure and the temperature for an incompressible flow. A detailed description of the physical background can be found in [1] and [2]. However, the well-posedness of the Bénard system is still an unsolved problem in the three-dimensional case. Therefore, it is particularly important to modify equations for Bénard system. We consider the following globally modified Bénard system:

$$
\left\{\begin{array}{lc}
\frac{\partial u}{\partial t}-v \Delta u+F_{N}(\|u\|)(u \cdot \nabla) u+\xi \omega+\nabla p=f, & \text { in }(0,+\infty) \times \Omega, \\
\frac{\partial \omega}{\partial t}-\Delta \omega+(u \cdot \nabla) \omega=g, & \text { in }(0,+\infty) \times \Omega, \\
\nabla \cdot u=0, & \text { in }(0,+\infty) \times \Omega, \\
u=0, \omega=0, & \text { on }(0,+\infty) \times \partial \Omega, \\
u(x, 0)=u_{0}(x), \omega(x, 0)=\omega_{0}(x), & x \in \Omega .
\end{array}\right.
$$

Here the function $F_{N}(r): \mathbb{R}^{+} \rightarrow \mathbb{R}^{+}$is defined by

$$
F_{N}(r):=\min \left\{1, \frac{N}{r}\right\}, r, N \in \mathbb{R}^{+} .
$$

In the modified term of Equation (2), $F_{N}(\|u\|)$ depends on the norm $\|u\|=\|\nabla u\|_{\left.L^{2}(\Omega)\right)^{3 \times 3}}$, which in turn depends on $\nabla u$ over the whole domain $\Omega$ and not just at or near the point $x \in \Omega$ under consideration. Essentially, it prevents large gradients dominating the dynamics and leading to explosions.

The globally modified Bénard system can be better described when we add the appropriate delay term to the system. In this way, it can take into account not only the present state of the system but the history of the solution. Namely, we consider the following equations:

$$
\left\{\begin{array}{lc}
\frac{\partial u}{\partial t}-v \Delta u+F_{N}(\|u\|)(u \cdot \nabla) u+\xi \omega+\nabla p=G_{1}(t, u(t-\rho(t))), & \text { in }(0,+\infty) \times \Omega, \\
\frac{\partial \omega}{\partial t}-\Delta \omega+(u \cdot \nabla) \omega=G_{2}(t, \omega(t-\rho(t))), & \text { in }(0,+\infty) \times \Omega, \\
\nabla \cdot u=0, & \text { in }(0,+\infty) \times \Omega, \\
u=0, \omega=0, & \text { on }(0,+\infty) \times \partial \Omega, \\
u(\tau, x)=u_{0}(x), \omega(\tau, x)=\omega_{0}(x), & x \in \Omega \\
u(t, x)=\phi_{1}(t-\tau, x), \omega(t, x)=\phi_{2}(t-\tau, x), & \text { in }(\tau-h, \tau) \times \Omega .
\end{array}\right.
$$

Here $\tau \in \mathbb{R}$ is the initial time, $G_{1}(t, u(t-\rho(t))), G_{2}(t, \omega(t-\rho(t)))$ are the external force terms which depends on $u(t-\rho(t)), \omega(t-\rho(t))$, respectively. $\rho(t) \geq 0$ is a delay function, $\phi_{1}, \phi_{2}$ are respectively the velocity, temperature defined on $(-h, 0)$. In addition, $\rho(t) \leq h, h>0$. When $\omega=0$, the Equation (3) is the Navier-Stokes equations with delays (see [3] [4]).

This paper is inspired by the literature [3]. The [3] studies mainly the existence and uniqueness of strong solutions and the asymptotic behavior of solutions for three-dimensional globally modified Navier-Stokes equations with delays under local Lipschitz conditions. The purpose of this paper is to study the existence, uniqueness and asymptotic behavior of solutions of three-dimensional 
globally modified Bénard system with delays under local Lipschitz conditions. This paper is organized as follows. In Section 2, we recall some definitions of function spaces and some related properties. In Section 3, we give the proof of the existence and uniqueness of weak solutions. In Section 4, we consider the asymptotic behavior of solutions.

\section{Preliminary}

Let

$$
\begin{aligned}
& \mathcal{V}=\left\{v \in\left(C_{0}^{\infty}(\Omega)\right)^{3}: \nabla \cdot v=0\right\} . \\
& H=c l_{\left(L^{2}(\Omega)\right)^{3}} \mathcal{V}, \quad V=c l_{\left(H_{0}^{1}(\Omega)\right)^{3}} \mathcal{V} .
\end{aligned}
$$

We denote by $(\cdot, \cdot)$ the scalar product, $\|\cdot\|_{2}$ the norm in $H$. For $u, v \in\left(L^{2}(\Omega)\right)^{3},(u, v)=\sum_{j=1}^{3} \int_{\Omega} u_{j}(x) v_{j}(x) \mathrm{d} x$. We denote by $((\cdot, \cdot))$ the scalar product, $\|\cdot\|$ the norm in $V$. For $u, v \in\left(H_{0}^{1}(\Omega)\right)^{3},((u, v))=\sum_{i, j=1}^{3} \int_{\Omega} \frac{\partial u_{j}}{\partial x_{i}} \frac{\partial v_{j}}{\partial x_{i}} \mathrm{~d} x$. The dual space of $V$ is $V^{\prime}$. It follows that $V \subset H \equiv H^{\prime} \subset V^{\prime}$, and the injections are dense and compact. We shall use $\|\cdot\|_{*}$ to denote the norm of $V^{\prime},\langle\cdot, \cdot\rangle$ represent the dual product between $V$ and $V^{\prime} . P$ is a Leray-Helmotz projection from $\left(L^{2}(\Omega)\right)^{3}$ to $H, \mathcal{T}$ is a Leray-Helmotz projection from $\left(L^{2}(\Omega)\right)^{3}$ to $L^{2}(\Omega)$, suppose $A_{1} u=-P \Delta u, A_{2} \omega=-\mathcal{T} \Delta \omega$, where $A_{1}, A_{2}$ are linear continuous operators. Let $D\left(A_{1}\right)$ be the domain of the operator $A_{1}$ in $H, D\left(A_{2}\right)$ be the domain of the operator $A_{2}$ in $L^{2}(\Omega)$. In addition,

$$
\left\langle A_{1} u, v\right\rangle_{V, V^{\prime}}=(\nabla u, \nabla v), \quad\left\langle A_{2} \omega, \eta\right\rangle_{H_{0}^{1}(\Omega), H^{-1}(\Omega)}=(\nabla \omega, \nabla \eta),
$$

where $u, v \in V, \omega, \eta \in H_{0}^{1}(\Omega)$.

Because of the regularity of the boundary $\partial \Omega$, it is easy to prove that: $D\left(A_{1}\right)=\left(H^{2}(\Omega)\right)^{3} \cap V, D\left(A_{2}\right)=H^{2}(\Omega) \cap H_{0}^{1}(\Omega)$, furthermore, we obtain

$$
D\left(A_{1}\right) \subset V \subset H, \quad D\left(A_{2}\right) \subset H_{0}^{1}(\Omega) \subset L^{2}(\Omega) .
$$

The first eigenvalue of Stokes operator is defined by $\lambda=\inf _{v \in V \backslash\{0\}} \frac{\|v\|^{2}}{\|\nu\|_{2}^{2}}>0$, let $\lambda_{1}$ be the minimum of the first eigenvalues of the operators $A_{1}, A_{2}$. For all $u, v, \omega \in V$, we put $b(u, v, \omega)=\int_{\Omega} \sum_{i, j=1}^{3} u_{i} \frac{\partial v_{j}}{\partial x_{i}} \omega_{j} \mathrm{~d} x$. We know from [1] that $b(u, v, \omega)$ is a trilinear continuous form on $V \times V \times V$, and $b(u, v, v)=0$ for $u \in V, v \in\left(H_{0}^{1}(\Omega)\right)^{3}$. For any $u, v, \omega \in V$, it follows that (see [5])

$$
|b(u, v, \omega)| \leq\left. C\|u\|\|v\||| \omega\right|^{\frac{1}{2}}\|\omega\|^{\frac{1}{2}} .
$$

Define

$$
b_{N}(u, v, w)=F_{N}(\|v\|) b(u, v, w), \forall u, v, w \in V,
$$

where $b_{N}$ is linear in $u, w$, but it is nonlinear in $v$. Obviously, $b_{N}(u, v, v)=0, \forall u, v \in V$. According to the property of $b$ (see [6]) and the defini- 
tion of $F_{N}$, we can get

$$
\left|b_{N}(u, v, \omega)\right| \leq N C_{1}\|u\|\|\omega\|, \forall u, v, \omega \in V .
$$

The following is a lemma, which is specifically proved in [5].

Lemma 2.1. For all $u, v \in V$ and $N>0$, the following two conclusions hold:

1) $\left.0 \leq\|u\| F_{N}(\|u\|) \leq N ; 2\right) \quad\left|F_{N}(\|u\|)-F_{N}(\|v\|)\right| \leq \frac{1}{N} F_{N}(\|u\|) F_{N}(\|v\|)\|u-v\|$.

For all $u \in V, v, \omega \in H_{0}^{1}(\Omega)$, let us define $c(u, v, \omega)=\int_{\Omega} \sum_{i=1}^{3} u_{i} \frac{\partial v}{\partial x_{i}} \omega \mathrm{d} x$. It has been given in [7], and $c(u, v, v)=0$ for $u \in V, v \in H_{0}^{1}(\Omega)$.

Let

$$
\begin{gathered}
\left\langle B_{N}(u, v), \omega\right\rangle=b_{N}(u, v, \omega), \forall u, v, \omega \in V, \\
\langle C(u, v), \omega\rangle=c(u, v, \omega), \forall u \in V, v, \omega \in H_{0}^{1}(\Omega) .
\end{gathered}
$$

The following describes the properties of the external force terms $G_{i}: \mathbb{R} \times H \rightarrow H, i=1,2:$

(c1) for any $u \in H, \omega \in L^{2}, G_{1}(\cdot, u): \mathbb{R} \rightarrow H, G_{2}(\cdot, \omega): \mathbb{R} \rightarrow L^{2}$ are measurable;

(c2) there exists nonnegative functions $g_{i} \in L_{l o c}^{p}(\mathbb{R}), i=1,2,1 \leq p \leq+\infty$, and a nondecreasing function $L:(0, \infty) \rightarrow(0, \infty)$, such that for all $R_{T_{i}}>0$, if $|u|,|v| \leq R_{T_{i}}$, then:

$$
\left|G_{i}(t, u)-G_{i}(t, v)\right| \leq L\left(R_{T_{i}}\right) g_{i}^{\frac{1}{2}}(t)|u-v|, i=1,2, t \in \mathbb{R}
$$

(c3) there exists nonnegative functions $f_{i} \in L_{l o c}^{1}(\mathbb{R}), i=1,2$, such that for any $u \in H \quad\left(\right.$ or $L^{2}$ ),

$$
\left|G_{i}(t, u)\right| \leq g_{i}(t)|u|^{2}+f_{i}(t), \forall t \in \mathbb{R}
$$

Supposing $\phi_{1} \in L^{2 p^{\prime}}(-h, 0 ; H), \phi_{2} \in L^{2 p^{\prime}}\left(-h, 0 ; L^{2}\right), \quad\left\{u_{0}, \omega_{0}\right\} \in H \times L^{2}$, where $\frac{1}{p}+\frac{1}{p^{\prime}}=1$. We consider a delay function $\rho \in C^{1}(\mathbb{R})$ for any $t \in \mathbb{R}$ such that $0 \leq \rho(t) \leq h$, and there exists a constant $\rho_{*}$ for any $t \in \mathbb{R}$ satisfying: $\rho^{\prime}(t) \leq \rho_{*}<1$.

We define $\tilde{g}_{i}(t)=g_{i} \circ \theta^{-1}(t)$, where $\theta(s)=s-\rho(s)$,

$\theta:[\tau,+\infty) \rightarrow[\tau-\rho(\tau),+\infty)$ is the differentiable and strictly increasing function, we obtain

$$
\begin{aligned}
& \int_{\tau}^{T}\left|G_{1}(t, u(t-\rho(t)))\right|^{2} \mathrm{~d} t \leq \int_{\tau}^{T} g_{1}(t)|u(t-\rho(t))|^{2} \mathrm{~d} t+\int_{\tau}^{T} f_{1}(t) \mathrm{d} t \\
& =\int_{\tau}^{T} \tilde{g}_{1}(t-\rho(t))|u(t-\rho(t))|^{2} \mathrm{~d} t+\int_{\tau}^{T} f_{1}(t) \mathrm{d} t \\
& =\int_{\tau-\rho(\tau)}^{T-\rho(T)} \tilde{g}_{1}(s)|u(s)|^{2} \cdot \frac{1}{1-\rho^{\prime}(s)} \mathrm{d} s+\int_{\tau}^{T} f_{1}(t) \mathrm{d} t \\
& \leq \frac{1}{1-\rho_{*}} \int_{\tau-\rho(\tau)}^{T-\rho(T)} \tilde{g}_{1}(t)|u(t)|^{2} \mathrm{~d} t+\int_{\tau}^{T} f_{1}(t) \mathrm{d} t \\
& \leq \frac{1}{1-\rho_{*}} \int_{\tau-\rho(\tau)}^{T} \tilde{g}_{1}(t)|u(t)|^{2} \mathrm{~d} t+\int_{\tau}^{T} f_{1}(t) \mathrm{d} t
\end{aligned}
$$


Similarly, when $i=2$, there is also an analogy estimate for the $\omega$ term.

Moreover, we call $\{u, \omega\} \in W_{T}$ as a set of solution for Equation (3) in $(0, T)$, when

$$
W_{T}=\left(L^{2}(0, T ; V) \cap L^{\infty}(0, T ; H)\right) \times\left(L^{2}\left(0, T ; H_{0}^{1}(\Omega)\right) \cap L^{\infty}\left(0, T ; L^{2}(\Omega)\right)\right),
$$

and for any $v \in V, \tilde{\omega} \in H_{0}^{1}(\Omega)$, the following is true:

$$
\left\{\begin{array}{l}
\frac{\mathrm{d}}{\mathrm{d} t}(u, v)+v((u, v))+b_{N}(u, u, v)+(\xi \omega, v)=\left(G_{1}(s, u(s-\rho(s))), v\right), \\
\frac{\mathrm{d}}{\mathrm{d} t}(\omega, \tilde{\omega})+((\omega, \tilde{\omega}))+c(u, \omega, \tilde{\omega})=\left(G_{2}(s, \omega(s-\rho(s))), \tilde{\omega}\right) .
\end{array}\right.
$$

\section{Existence and Uniqueness of Weak Solutions}

Theorem 3.1. Under the conditions (c1)-(c3), assume that $\tau \in \mathbb{R}^{+}$, $\left\{u_{0}, \omega_{0}\right\} \in H \times L^{2}$ and $\phi_{1} \in L^{2 p^{\prime}}(-h, 0 ; H), \phi_{2} \in L^{2 p^{\prime}}\left(-h, 0 ; L^{2}\right)$. Then there exists a weak solution $\{u, \omega\}$ of equation (3).

Proof. For simplicity, and without loss of generality, we assume $\tau=0$. We consider the proof of the existence of weak solutions of Equation (3). We use Galerkin method to prove the existence of solutions, which is standard (see [8] [9]).

Consider the following equations:

$$
\left\{\begin{array}{l}
\frac{\partial u_{m}}{\partial t}+v A_{1} u_{m}+P_{m} B_{N}\left(u_{m}, u_{m}\right)+\xi \omega_{m}=P_{m} G_{1}\left(t, u_{m}(t-\rho(t))\right), \\
\frac{\partial \omega_{m}}{\partial t}+A_{2} \omega_{m}+\mathcal{T}_{m} C\left(u_{m}, \omega_{m}\right)=\mathcal{T}_{m} G_{2}\left(t, \omega_{m}(t-\rho(t))\right), \\
u_{m}(0)=P_{m} u_{0}, \omega_{m}(0)=\mathcal{T}_{m} \omega_{0}, \\
u_{m}=P_{m} \phi_{1}, \omega_{m}=\mathcal{T}_{m} \phi_{2}, u_{m}, \omega_{m} \in(-h, 0) .
\end{array}\right.
$$

Next, we need to make some priori estimates for the approximate solution $\left\{u_{m}, \omega_{m}\right\}$. So taking the inner product of the second equation of (7) with $\omega_{m}$, and using $c\left(u_{m}, \omega_{m}, \omega_{m}\right)=0$, we obtain

$$
\begin{aligned}
\frac{1}{2} \frac{\mathrm{d}}{\mathrm{d} t}\left|\omega_{m}(t)\right|^{2}+\|\omega(t)\|^{2} & =\left(G_{2}\left(t, \omega_{m}(t-\rho(t))\right), \omega_{m}\right) \\
& \leq \frac{1}{2 \lambda_{1}}\left|G_{2}\left(t, \omega_{m}(t-\rho(t))\right)\right|^{2}+\frac{\lambda_{1}}{2}\left|\omega_{m}(t)\right|^{2} \\
& \leq \frac{1}{2 \lambda_{1}}\left|G_{2}\left(t, \omega_{m}(t-\rho(t))\right)\right|^{2}+\frac{1}{2}\left\|\omega_{m}(t)\right\|^{2},
\end{aligned}
$$

that is

$$
\frac{\mathrm{d}}{\mathrm{d} t}\left|\omega_{m}\right|^{2}+\left\|\omega_{m}\right\|^{2} \leq \frac{1}{\lambda_{1}}\left|G_{2}\left(t, \omega_{m}(t-\rho(t))\right)\right|^{2} .
$$

Integrating over $(0, t)$, and using (6), we obtain for $t \in\left[0, T_{2}\right]$

$$
\begin{aligned}
& \left|\omega_{m}(t)\right|^{2}+\int_{0}^{t}\left\|\omega_{m}(s)\right\|^{2} \mathrm{~d} s \\
& \leq\left|\omega_{0}\right|^{2}+\frac{1}{\lambda_{1}} \int_{0}^{t}\left|G_{2}\left(s, \omega_{m}(s-\rho(s))\right)\right|^{2} \mathrm{~d} s \\
& \leq K_{T_{2}}+\frac{1}{\lambda_{1}\left(1-\rho_{*}\right)} \int_{0}^{t} \tilde{g}_{2}(s)\left|\omega_{m}(s)\right|^{2} \mathrm{~d} s,
\end{aligned}
$$


where $K_{T_{2}}=\left|\omega_{0}\right|^{2}+\frac{1}{\lambda_{1}\left(1-\rho_{*}\right)} \int_{-\rho(0)}^{0} \tilde{g}_{2}(s)\left|\phi_{2}(s)\right|^{2} \mathrm{~d} s+\frac{1}{\lambda_{1}} \int_{0}^{T_{2}} f_{2}(s) \mathrm{d} s$. It follows from Gronwall lemma that

$$
\left|\omega_{m}(t)\right|^{2} \leq K_{T_{2}} \exp \left\{\frac{1}{\lambda_{1}\left(1-\rho_{*}\right)} \int_{0}^{T_{2}} \tilde{g}_{2}(s) \mathrm{d} s\right\}=C_{T_{2}}, \forall t \in\left[0, T_{2}\right] .
$$

Taking the inner product of the first equation of (7) with $u_{m}$, from (9) and $b_{N}\left(u_{m}, u_{m}, u_{m}\right)=0$, we obtain

$$
\begin{aligned}
& \frac{1}{2} \frac{\mathrm{d}}{\mathrm{d} t}\left|u_{m}(t)\right|^{2}+v \|\left. u_{m}(t)\right|^{2} \\
& \leq|\xi|\left(\omega_{m}(t), u_{m}(t)\right)+\left(G_{1}\left(t, u_{m}(t-\rho(t))\right), u_{m}\right) \\
& \leq \frac{1}{v \lambda_{1}} \xi^{2}\left|\omega_{m}(t)\right|^{2}+\frac{v \lambda_{1}}{4}\left|u_{m}(t)\right|^{2}+\frac{1}{v \lambda_{1}}\left|G_{1}\left(t, u_{m}(t-\rho(t))\right)\right|^{2}+\frac{v \lambda_{1}}{4}\left|u_{m}(t)\right|^{2} \\
& \leq \frac{1}{v \lambda_{1}}\left|G_{1}\left(t, u_{m}(t-\rho(t))\right)\right|^{2}+\frac{v}{2}\left\|u_{m}(t)\right\|^{2}+\frac{\xi^{2}}{v \lambda_{1}} C_{T_{2}},
\end{aligned}
$$

that is

$$
\frac{\mathrm{d}}{\mathrm{d} t}\left|u_{m}\right|^{2}+v\left\|u_{m}\right\|^{2} \leq \frac{2}{v \lambda_{1}}\left|G_{1}\left(t, u_{m}(t-\rho)(t)\right)\right|^{2}+\frac{2 \xi^{2}}{v \lambda_{1}} C_{T_{2}} .
$$

Integrating over $(0, t)$, and using (6), we obtain for $t \in\left[0, T_{1}\right]$

$$
\begin{aligned}
& \left|u_{m}(t)\right|^{2}+\left.v \int_{0}^{t}|| u_{m}(s)\right|^{2} \mathrm{~d} s \\
& \leq\left|u_{0}\right|^{2}+\frac{2}{v \lambda_{1}} \int_{0}^{t}\left|G_{1}\left(s, u_{m}(s-\rho(s))\right)\right|^{2} \mathrm{~d} s+\frac{2 \xi^{2}}{v \lambda_{1}} \int_{0}^{t} C_{T_{2}} \mathrm{~d} s \\
& \leq K_{T_{1}}+\frac{2}{v \lambda_{1}\left(1-\rho_{*}\right)} \int_{0}^{t} \tilde{g}_{1}(s)\left|u_{m}(s)\right|^{2} \mathrm{~d} s,
\end{aligned}
$$

where

$K_{T_{1}}=\left|u_{0}\right|^{2}+\frac{2 \xi^{2}}{v \lambda_{1}} \int_{0}^{T_{1}} C_{T_{2}} \mathrm{~d} s+\frac{2}{v \lambda_{1}\left(1-\rho_{*}\right)} \int_{-\rho(0)}^{0} \tilde{g}_{1}(s)\left|\phi_{1}(s)\right|^{2} \mathrm{~d} s+\frac{2}{v \lambda_{1}} \int_{0}^{T_{1}} f_{1}(s) \mathrm{d} s \quad . \quad$ It follows from Gronwall lemma that

$$
\left|u_{m}(t)\right|^{2} \leq K_{T_{1}} \exp \left\{\frac{2}{v \lambda_{1}\left(1-\rho_{*}\right)} \int_{0}^{T_{1}} \tilde{g}_{1}(s) \mathrm{d} s\right\}=C_{T_{1}}, \forall t \in\left[0, T_{1}\right] .
$$

From (10) and (11), a subsequence of $\left\{u_{m}\right\}$ is bounded in $L^{\infty}(0, T ; H)$ and $L^{2}(0, T ; V)$ (where $T=\max \left\{T_{1}, T_{2}\right\}$ ). Next from the first equation of (7) and (5), we obtain that $\frac{\mathrm{d} u_{m}}{\mathrm{~d} t}$ is bounded in $L^{2}\left(0, T ; V^{\prime}\right)$. Therefore, there exists an element $u \in L^{\infty}(0, T ; H) \cap L^{2}(0, T ; V)$ and $\frac{\mathrm{d} u}{\mathrm{~d} t} \in L^{2}\left(0, T ; V^{\prime}\right)$, such that

$$
\left\{\begin{array}{l}
u_{m} \stackrel{*}{\rightarrow} u \text { in } L^{\infty}(0, T ; H), \\
u_{m} \rightarrow u \text { in } L^{2}(0, T ; V), \\
\frac{\mathrm{d} u_{m}}{\mathrm{~d} t} \rightarrow \frac{\mathrm{d} u}{\mathrm{~d} t} \text { in } L^{2}\left(0, T ; V^{\prime}\right) .
\end{array}\right.
$$


Obviously, $u \in C([0,+\infty) ; H)$ (see [10]). By the compactness Aubin-Lions (see [9]), we can deduce

$$
\left\{\begin{array}{l}
u_{m} \rightarrow u \text { in } L^{2}(0, T ; H), \\
u_{m} \rightarrow u \text { a.e.in }(0, T) \times \Omega .
\end{array}\right.
$$

Similarly, from (8) and (9), there is also an analogy conclusion for the $\omega_{m}$ term.

In summary, when $t_{m}=+\infty, m \geq 1$, there exists $\{u, \omega\} \in\left(L^{\infty}(0, T ; H) \cap L^{2}(0, T ; V)\right) \times\left(L^{\infty}\left(0, T ; L^{2}(\Omega)\right) \cap L^{2}\left(0, T ; H_{0}^{1}(\Omega)\right)\right.$ for any $T>0$, such that

$$
\left\{\begin{array}{l}
\left\{u_{m}, \omega_{m}\right\} \rightarrow\{u, \omega\} \text { in } L^{2}(0, T ; H) \times L^{2}\left(0, T ; L^{2}(\Omega)\right), \\
\left\{u_{m}, \omega_{m}\right\} \rightarrow\{u, \omega\} \text { in } H \times L^{2}(\Omega) \text { a.e.t } \in(0, T) .
\end{array}\right.
$$

Since $\left\{u_{m}, \omega_{m}\right\}$ weak converges to $\{u, \omega\}$ in $V \times H_{0}^{1}(\Omega)$, we cannot deduce that $\left\|u_{m}\right\| \rightarrow\|u\|,\left\|\omega_{m}\right\| \rightarrow\|\omega\|$ or $F_{N}\left(\left\|u_{m}(t)\right\|\right) \rightarrow F_{N}(\|u(t)\|)$ and $C\left(u_{m}, \omega_{m}\right) \rightarrow C(u, \omega)$ at least almost everywhere. So we need make a stronger estimate.

Taking the inner product of the first equation of (7) with $A_{1} u_{m}$, and using (9), we obtain

$$
\begin{aligned}
& \frac{1}{2} \frac{\mathrm{d}}{\mathrm{d} t}\left\|u_{m}\right\|^{2}+v\left|A_{1} u_{m}\right|^{2} \\
\leq & b_{N}\left(u_{m}, u_{m}, A_{1} u_{m}\right)+|\xi|\left(\omega_{m}, A_{1} u_{m}\right)+\left(G_{1}\left(t, u_{m}(t-\rho(t))\right), A_{1} u_{m}\right) \\
\leq & \left.\frac{N}{\mid u_{m} \|} C\left|u_{m}\right|\right|^{\frac{3}{2}}\left|A_{1} u_{m}\right|^{\frac{3}{2}}+|\xi|\left(\omega_{m}, A_{1} u_{m}\right)+\left(G_{1}\left(t, u_{m}(t-\rho(t))\right), A_{1} u_{m}\right) \\
\leq & C N^{4}\left\|u_{m}\right\|^{2}+\frac{v}{8}\left|A_{1} u_{m}\right|^{2}+\frac{1}{v} \xi^{2}\left|\omega_{m}\right|^{2}+\frac{v}{4}\left|A_{1} u_{m}\right|^{2} \\
& +\frac{2}{v}\left|G_{1}\left(t, u_{m}(t-\rho(t))\right)\right|^{2}+\frac{v}{8}\left|A_{1} u_{m}\right|^{2} \\
\leq & \frac{v}{2}\left|A_{1} u_{m}\right|^{2}+C N^{4}|| u_{m} \|^{2}+\frac{\xi^{2}}{v} C_{T_{2}}+\frac{2}{v}\left|G_{1}\left(t, u_{m}(t-\rho(t))\right)\right|^{2},
\end{aligned}
$$

that is

$$
\frac{\mathrm{d}}{\mathrm{d} t}\left\|u_{m}\right\|^{2}+v\left|A_{1} u_{m}\right|^{2} \leq C N^{4}\left\|u_{m}\right\|^{2}+\frac{2 \xi^{2}}{v} C_{T_{2}}+\frac{4}{v}\left|G_{1}\left(t, u_{m}(t-\rho(t))\right)\right|^{2} .
$$

Integrating over $(s, t)$, and using (6), we obtain for all $0 \leq s \leq t \leq T$

$$
\begin{aligned}
& \left\|u_{m}(t)\right\|^{2} \\
& \leq\left\|u_{m}(s)\right\|^{2}+C N^{4} \int_{0}^{T}\left\|u_{m}(r)\right\|^{2} \mathrm{~d} r+\frac{2 \xi^{2}}{v} \int_{0}^{T} C_{T_{2}} \mathrm{~d} r+\frac{4}{v} \int_{0}^{T}\left|G_{1}\left(r, u_{m}(r-\rho(r))\right)\right|^{2} \mathrm{~d} r \\
& \leq\left\|u_{m}(s)\right\|^{2}+C N^{4} \int_{0}^{T}\left\|u_{m}(r)\right\|^{2} \mathrm{~d} r+\frac{2 \xi^{2}}{v} \int_{0}^{T} C_{T_{2}} \mathrm{~d} r+\frac{4}{v\left(1-\rho_{*}\right)} \int_{-\rho(0)}^{0} \tilde{g}_{1}(r)\left|\phi_{1}(r)\right|^{2} \mathrm{~d} r(13) \\
& \\
& +\frac{4}{v\left(1-\rho_{*}\right)} \int_{0}^{T} \tilde{g}_{1}(r)\left|u_{m}(r)\right|^{2} \mathrm{~d} r+\frac{4}{v} \int_{0}^{T} f_{1}(r) \mathrm{d} r \\
& :=\left\|u_{m}(s)\right\|^{2}+\Phi .
\end{aligned}
$$


The following estimate $\Phi$. Thanks to (10) and (11), we know that $u_{m}$ is bounded in $L^{2}(0, T ; V) \cap L^{\infty}(0, T ; H)$, therefore, there exists $\tilde{K}_{T_{1}}>0$, such that for all integer $m \geq 1$

$$
\begin{aligned}
& C N^{4} \int_{0}^{T}\left\|u_{m}(r)\right\|^{2} \mathrm{~d} r+\frac{2 \xi^{2}}{v} \int_{0}^{T} C_{T_{2}} \mathrm{~d} r+\frac{4}{v\left(1-\rho_{*}\right)} \int_{-\rho(0)}^{0} \tilde{g}_{1}(r)\left|\phi_{1}(r)\right|^{2} \mathrm{~d} r \\
& +\frac{4}{v\left(1-\rho_{*}\right)} \int_{0}^{T} \tilde{g}_{1}(r)\left|u_{m}(r)\right|^{2} \mathrm{~d} r+\frac{4}{v} \int_{0}^{T} f_{1}(r) \mathrm{d} r \leq \tilde{K}_{T_{1}} .
\end{aligned}
$$

Integrating (13) for $s$ over $[0, t]$, we obtain

$$
t\left\|u_{m}(t)\right\|^{2} \leq \int_{0}^{T}\left\|u_{m}(s)\right\|^{2} \mathrm{~d} s+T \tilde{K}_{T_{1}} \leq \sup _{m \geq 1}\left(\int_{0}^{T}\left\|u_{m}(s)\right\|^{2} \mathrm{~d} s\right)+T \tilde{K}_{T_{1}}:=\hat{K}_{T_{1}} .
$$

Consequently, $\left\|u_{m}(t)\right\|^{2} \leq \frac{1}{\varepsilon} \hat{K}_{T_{1}}$ for any $t \in[\varepsilon, T]$ and any $0<\varepsilon<T$.

On the other hand, using the uniformly Gronwall inequality for (12), we have

$$
\left\|u_{m}\right\|^{2} \leq\left(\frac{\tilde{K}_{T_{1}}}{C N^{4}}+\frac{2 \xi^{2}}{v} C_{T_{2}}+\frac{4}{v}\left|G_{1}\left(t, u_{m}(t-\rho(t))\right)\right|^{2}\right) \mathrm{e}^{C N^{4}}=C_{T_{1}}^{\prime} .
$$

Taking the inner product of the second equation of (7) with $A_{2} \omega_{m}$, we obtain

$$
\begin{aligned}
& \frac{1}{2} \frac{\mathrm{d}}{\mathrm{d} t}\left\|\omega_{m}\right\|^{2}+\left|A_{2} \omega_{m}\right|^{2} \\
& \leq c\left(u_{m}, \omega_{m}, A_{2} \omega_{m}\right)+\left(G_{2}\left(t, \omega_{m}(t-\rho(t))\right), A_{2} \omega_{m}\right) \\
& \leq C\left\|u_{m}||^{2}\right\| \omega_{m} \|^{2}+\frac{1}{4}\left|A_{2} \omega_{m}\right|^{2}+\left|G_{2}\left(t, \omega_{m}(t-\rho(t))\right)\right|^{2}+\frac{1}{4}\left|A_{2} \omega_{m}\right|^{2} \\
& \leq C C_{T_{1}}^{\prime}\left\|\omega_{m}\right\|^{2}+\frac{1}{4}\left|A_{2} \omega_{m}\right|^{2}+\left|G_{2}\left(t, \omega_{m}(t-\rho(t))\right)\right|^{2}+\frac{1}{4}\left|A_{2} \omega_{m}\right|^{2},
\end{aligned}
$$

where

$$
c\left(u, \omega, A_{2} \omega\right) \leq\left.|u|_{\infty}\left|\nabla \omega\left\|A_{2} \omega|\leq C|\right\| u\|\| \omega\left\|\left|A_{2} \omega\right| \leq C\right\| u\left\|^{2}\right\| \omega \|^{2}+\frac{1}{4}\right| A_{2} \omega\right|^{2} .
$$

That is

$$
\frac{\mathrm{d}}{\mathrm{d} t}\left\|\omega_{m}\right\|^{2}+\left|A_{2} \omega_{m}\right|^{2} \leq C C_{T_{1}}^{\prime}\left\|\omega_{m}\right\|^{2}+2\left|G_{2}\left(t, \omega_{m}(t-\rho(t))\right)\right|^{2} .
$$

Similarly, integrating over $(s, t)$, we obtain for all $0 \leq s \leq t \leq T$

$$
\left\|\omega_{m}(t)\right\|^{2} \leq\left\|\omega_{m}(s)\right\|^{2}+\tilde{K}_{T_{2}},
$$

where

$$
\begin{aligned}
& C C_{T_{1}}^{\prime} \int_{0}^{T}\left\|\omega_{m}(r)\right\|^{2} \mathrm{~d} r+\frac{2}{1-\rho_{*}} \int_{-\rho(0)}^{0} \tilde{g}_{2}(r)\left|\phi_{2}(r)\right|^{2} \mathrm{~d} r \\
& +\frac{2}{1-\rho_{*}} \int_{0}^{T} \tilde{g}_{2}(r)\left|\omega_{m}(r)\right|^{2} \mathrm{~d} r+2 \int_{0}^{T} f_{1}(r) \mathrm{d} r \leq \tilde{K}_{T_{2}} .
\end{aligned}
$$

Integrating (15) for $s$ over $[0, t]$, we obtain

$$
t\left\|\omega_{m}(t)\right\|^{2} \leq \sup _{m \geq 1}\left(\int_{0}^{T}\left\|\omega_{m}(s)\right\|^{2} \mathrm{~d} s\right)+T \tilde{K}_{T_{2}}:=\hat{K}_{T_{2}} .
$$

Consequently, $\left\|\omega_{m}(t)\right\|^{2} \leq \frac{1}{\varepsilon} \hat{K}_{T_{2}}$ for all $t \in[\varepsilon, T]$ and all $0<\varepsilon<T$. 
On the other hand, using the uniformly Gronwall inequality for (14), we have

$$
\left\|\omega_{m}\right\|^{2} \leq\left(\frac{\tilde{K}_{T_{2}}}{C C_{T_{1}}^{\prime}}+\left|G_{2}\left(t, \omega_{m}(t-\rho(t))\right)\right|^{2}\right) \mathrm{e}^{C C_{T_{1}}^{\prime}}=C_{T_{2}}^{\prime} .
$$

From the above discussion, we can deduce that $u_{m}$ is bounded in $L^{\infty}(\varepsilon, T ; V)$ and $L^{2}\left(\varepsilon, T ; D\left(A_{1}\right)\right)$. What's more, when $u_{0} \in V$, owing to $\left\|u_{m}(0)\right\| \ll\left\|P_{m} u_{0}\right\|$ $\leq\left\|u_{0}\right\|$, we deduce that $u_{m}$ is bounded in $L^{\infty}(0, T ; V) \cap L^{2}\left(0, T ; D\left(A_{1}\right)\right)$; when $u_{0} \in H$, it follows that $D\left(A_{1}\right) \subset V \subset H$ and injections are compact. Supposing there exists a positive sequence $\left\{\varepsilon_{n}\right\} \searrow 0$, such that

$$
u \in L^{\infty}(0, T ; H) \cap L^{2}(0, T ; V) \cap L^{\infty}(\varepsilon, T ; V) \cap L^{2}\left(\varepsilon, T ; D\left(A_{1}\right)\right) .
$$

Therefore

$$
\left\{\begin{array}{l}
u_{m} \stackrel{*}{\rightarrow} \text { in } L^{\infty}(0, T ; V) \\
u_{m} \rightarrow u \text { in } L^{2}\left(0, T ; D\left(A_{1}\right)\right) \\
u_{m} \rightarrow u \text { in } L^{\infty}(0, T ; V) \\
u_{m} \rightarrow u \text { in }(0, T) \times \Omega .
\end{array}\right.
$$

As long as there exists $u_{m} \rightarrow u$, in $L^{2}(\varepsilon, T ; V)$ for $T>\varepsilon>0$, we can get

$$
\begin{aligned}
\left\|u_{m}\right\| & \rightarrow\|u\| \text { a.e.in }(0,+\infty), \\
F_{N}\left(\left\|u_{m}(t)\right\|\right) & \rightarrow F_{N}(\|u(t)\|) \text { a.e.in }(0,+\infty) .
\end{aligned}
$$

Similarly, the same is true for the $\omega_{m}$ term. In conclusion, $\{u, \omega\}$ is a set of solution for Equation (3).

Theorem 3.2. Under the previous all assumptions, there exists a unique solution of equations (3).

Proof. Let $\left\{u_{1}, \omega_{1}\right\}$ and $\left\{u_{2}, \omega_{2}\right\}$ be two solutions of equations (3), and set $\{u, \omega\}=\left\{u_{1}-u_{2}, \omega_{1}-\omega_{2}\right\}$, according to the first equation of (3), we obtain

$$
\begin{aligned}
& \frac{1}{2} \frac{\mathrm{d}}{\mathrm{d} t}|u|^{2}+v\|u\|^{2}+\left\langle\mathcal{N} \mathcal{L}\left(u_{1}, u_{2}\right), u\right\rangle+\xi(\omega, u) \\
& =\left(G_{1}\left(t, u_{1}(t-\rho(t))\right)-G_{1}\left(t, u_{2}(t-\rho(t))\right), u\right),
\end{aligned}
$$

where $\left\langle\mathcal{N} \mathcal{L}\left(u_{1}, u_{2}\right), u\right\rangle=F_{N}\left(\left\|u_{1}\right\|\right) b\left(u_{1}, u_{1}, u\right)-F_{N}\left(\left\|u_{2}\right\|\right) b\left(u_{2}, u_{2}, u\right)$, using the property of trilinear form $b$, we easily get

$$
\begin{aligned}
& \left\langle\mathcal{N} \mathcal{L}\left(u_{1}, u_{2}\right), u\right\rangle \\
& =F_{N}\left(\left\|u_{1}\right\|\right) b\left(u, u_{1}, u\right)+\left(F_{N}\left(\left\|u_{1}\right\|\right)-F_{N}\left(\left\|u_{2}\right\|\right)\right) b\left(u_{2}, u_{1}, u\right)+F_{N}\left(\left\|u_{2}\right\|\right) b\left(u_{2}, u, u\right) .
\end{aligned}
$$

From Lemma 2.1, (4) and the Young inequality, we obtain

$$
\left\langle\mathcal{N L}\left(u_{1}, u_{2}\right), u\right\rangle \leq \frac{v}{2}\|u\|^{2}+C N^{4}|u|^{2}
$$

Therefore

$$
\begin{aligned}
& \frac{1}{2} \frac{\mathrm{d}}{\mathrm{d} t}|u|^{2}+\frac{v}{2} \|\left. u\right|^{2} \\
& \leq C N^{4}|u|^{2}+\frac{\xi^{2}}{2}|\omega|^{2}+\frac{1}{2}|u|^{2}+\left(G_{1}\left(t, u_{1}(t-\rho(t))\right)-G_{1}\left(t, u_{2}(t-\rho(t))\right), u\right)
\end{aligned}
$$


To estimate on the last term for above formula. For fixed $T_{1}>\tau$, $u_{i} \in C\left(\left[\tau, T_{1}\right] ; H\right)$, there exists $s \in\left[\tau, T_{1}\right]$, such that $\left|u_{i}(s)\right| \leq R_{T_{1}}$, in addition, using (c2), $u=0, t \in(\tau-h, \tau)$, the Hölder inequality, Young's inequality and (6), we obtain for any $t \in\left[\tau, T_{1}\right]$

$$
\begin{aligned}
& \int_{\tau}^{T}\left(G_{1}\left(s, u_{1}(s-\rho(s))\right)-G_{1}\left(s, u_{2}(s-\rho(s))\right), u\right) \mathrm{d} s \\
& \leq \int_{\tau}^{T}\left|G_{1}\left(s, u_{1}(s-\rho(s))\right)-G_{1}\left(s, u_{2}(s-\rho(s))\right)\right||u(s)| \mathrm{d} s \\
& \leq L\left(R_{T_{1}}\right) \int_{\tau}^{T} g_{1}^{\frac{1}{2}}(s)|u(s-\rho(s))||u(s)| \mathrm{d} s \\
& \leq L\left(R_{T_{1}}\right)\left[\int_{\tau}^{T} \frac{\left(1-\rho_{*}\right)^{\frac{1}{2}}}{2} g_{1}(s)|u(s-\rho(s))|^{2} \mathrm{~d} s+\int_{\tau}^{T} \frac{1}{2\left(1-\rho_{*}\right)^{\frac{1}{2}}}|u(s)|^{2} \mathrm{~d} s\right] \\
& \leq L\left(R_{T_{1}}\right)\left[\frac{\left(1-\rho_{*}\right)^{\frac{1}{2}}}{2} \cdot \frac{1}{1-\rho_{*}} \int_{\tau-\rho(\tau)}^{T} \tilde{g}_{1}(s)|u(s)|^{2} \mathrm{~d} s+\frac{1}{2\left(1-\rho_{*}\right)^{\frac{1}{2}}} \int_{\tau}^{T}|u(s)|^{2} \mathrm{~d} s\right] \\
& =\frac{L\left(R_{T_{1}}\right)}{2\left(1-\rho_{*}\right)^{\frac{1}{2}}} \int_{\tau}^{T}\left(\tilde{g}_{1}(s)+1\right)|u(s)|^{2} \mathrm{~d} s .
\end{aligned}
$$

Therefore

$$
\frac{1}{2} \frac{\mathrm{d}}{\mathrm{d} t}|u|^{2}+\frac{v}{2}\|u\|^{2} \leq C N^{4}|u|^{2}+\frac{\xi^{2}}{2}|\omega|^{2}+\frac{1}{2}|u|^{2}+\frac{L\left(R_{T_{1}}\right)}{2\left(1-\rho_{*}\right)^{\frac{1}{2}}}\left(\tilde{g}_{1}(t)+1\right)|u|^{2} .
$$

Similarly, according to the second equation of (3), we obtain

$$
\begin{aligned}
& \frac{1}{2} \frac{\mathrm{d}}{\mathrm{d} t}|\omega|^{2}+\|\omega\|^{2}+c\left(u_{1}, \omega_{1}, \omega\right)-c\left(u_{2}, \omega_{2}, \omega\right) \\
& =\left(G_{2}\left(t, \omega_{1}(t-\rho(t))\right)-G_{2}\left(t, \omega_{2}(t-\rho(t))\right), \omega\right) .
\end{aligned}
$$

Since

$$
\begin{aligned}
c\left(u_{1}, \omega_{1}, \omega\right)-c\left(u_{2}, \omega_{2}, \omega\right) & =c\left(u, \omega_{1}, \omega\right)+c\left(u_{2}, \omega, \omega\right) \\
& \leq|u|_{\infty}\left|\nabla \omega_{1}\right||\omega|+\left|u_{2}\right||\nabla \omega|_{\infty}|\omega| \\
& \leq C\|u\|\left|\omega_{1}\left\||\omega|+C\left|u_{2}\right|\right\| \omega \|\right| \omega \mid \\
& \leq \frac{v}{2}\|u\|^{2}+C\left\|\omega_{1}\right\|^{2}|\omega|^{2}+\|\omega\|^{2}+C\left|u_{2}\right|^{2}|\omega|^{2} \\
& \leq \frac{v}{2}\|u\|^{2}+C\left(C_{T_{2}}^{\prime}+C_{T_{1}}\right)|\omega|^{2}+\|\omega\|^{2},
\end{aligned}
$$

where (16) is used, we can get

$$
\begin{aligned}
& \frac{1}{2} \frac{\mathrm{d}}{\mathrm{d} t}|\omega|^{2} \\
& \leq \frac{v}{2}\|u\|^{2}+C\left(C_{T_{2}}^{\prime}+C_{T_{1}}\right)|\omega|^{2}+\left(G_{2}\left(t, \omega_{1}(t-\rho(t))\right)-G_{2}\left(t, \omega_{2}(t-\rho(t))\right), \omega\right) \\
& \leq \frac{v}{2}\|u\|^{2}+C\left(C_{T_{2}}^{\prime}+C_{T_{1}}\right)|\omega|^{2}+\frac{L\left(R_{T_{2}}\right)}{2\left(1-\rho_{*}\right)^{\frac{1}{2}}}\left(\tilde{g}_{2}(t)+1\right)|\omega|^{2} .
\end{aligned}
$$


From (17) and (18), we obtain

$$
\begin{aligned}
\frac{\mathrm{d}}{\mathrm{d} t}\left(|u|^{2}+|\omega|^{2}\right) \leq & {\left[\left(C N^{4}+1\right)+\frac{L\left(R_{T_{1}}\right)}{\left(1-\rho_{*}\right)^{\frac{1}{2}}}\left(\tilde{g}_{1}(t)+1\right)\right]|u|^{2} } \\
& +\left[C\left(C_{T_{2}}^{\prime}+C_{T_{1}}+\xi^{2}\right)+\frac{L\left(R_{T_{2}}\right)}{\left(1-\rho_{*}\right)^{\frac{1}{2}}}\left(\tilde{g}_{2}(t)+1\right)\right]|\omega|^{2} .
\end{aligned}
$$

Because the coefficients of $|u|^{2}$ and $|\omega|^{2}$ are bounded at the right of the equation, the conclusion follows from Gronwall lemma, since $|u(0)|^{2}=0,|\omega(0)|^{2}=0$.

\section{Asymptotic Behavior of Solutions}

In this section, we prove mainly the asymptotic behavior of the solution of Equations (3) when $t \rightarrow \infty$.

Suppose that (c1)-(c2) hold with $g_{i} \in L^{\infty}(\mathbb{R})$, assume also that: $v^{2} \lambda_{1}^{2}\left(1-\rho_{*}\right)>2\left|g_{1}\right|_{\infty}, \quad \lambda_{1}^{2}\left(1-\rho_{*}\right)>\left|g_{2}\right|_{\infty}$, where $\left|g_{i}\right|_{\infty}:=\left\|g_{i}\right\|_{L^{\infty}(\mathbb{R})}$, and let us denote respectively by $\varepsilon_{1}, \varepsilon_{2}>0$ the unique solution of

$$
\begin{gathered}
\varepsilon_{1}-v \lambda_{1}+\frac{2\left|g_{1}\right|_{\infty} \mathrm{e}^{\varepsilon_{1} h}}{v \lambda_{1}\left(1-\rho_{*}\right)}=0, \\
\varepsilon_{2}-\lambda_{1}+\frac{\left|g_{2}\right|_{\infty} \mathrm{e}^{\varepsilon_{2} h}}{\lambda_{1}\left(1-\rho_{*}\right)}=0 .
\end{gathered}
$$

Theorem 4.1. Under the above assumptions, for any $\left\{u_{0}, \omega_{0}\right\} \in H \times L^{2}$, $\phi_{1} \in L^{2}(-h, 0 ; H), \phi_{2} \in L^{2}\left(-h, 0 ; L^{2}\right)$ and any $\tau \in \mathbb{R}$, the solution $\left\{u\left(t ; \tau, u_{0}, \phi_{1}\right), \omega\left(t ; \tau, \omega_{0}, \phi_{2}\right)\right\}$ of equations (3) satisfies

$$
\begin{aligned}
\left|u\left(t ; \tau, u_{0}, \phi_{1}\right)\right|^{2} \leq & \left(\left|u_{0}\right|^{2}+\frac{2\left|g_{1}\right|_{\infty} \mathrm{e}^{\varepsilon_{1} h}}{v \lambda_{1}\left(1-\rho_{*}\right)} \int_{-h}^{0} \mathrm{e}^{\varepsilon_{1} s}\left|\phi_{1}(s)\right|^{2} \mathrm{~d} s\right) \mathrm{e}^{\varepsilon_{1}(\tau-t)} \\
& +\mathrm{e}^{-\varepsilon_{1} t}\left(\frac{2}{v \lambda_{1}} \int_{\tau}^{t} \mathrm{e}^{\varepsilon_{1} s} f_{1}(s) \mathrm{d} s+\frac{2 \xi^{2}}{v \lambda_{1}} \int_{\tau}^{t} \mathrm{e}^{\varepsilon_{1} s} C_{T_{2}} \mathrm{~d} s\right) \\
\left|\omega\left(t ; \tau, \omega_{0}, \phi_{2}\right)\right|^{2} \leq & \left(\left|\omega_{0}\right|^{2}+\frac{\left|g_{2}\right|_{\infty} \mathrm{e}^{\varepsilon_{2} h}}{\lambda_{1}\left(1-\rho_{*}\right)} \int_{-h}^{0} \mathrm{e}^{\varepsilon_{2} s}\left|\phi_{2}(s)\right|^{2} \mathrm{~d} s\right) \mathrm{e}^{\varepsilon_{2}(\tau-t)} \\
& +\frac{1}{\lambda_{1}} \mathrm{e}^{-\varepsilon_{2} t} \int_{\tau}^{t} \mathrm{e}^{\varepsilon_{2} s} f_{2}(s) \mathrm{d} s .
\end{aligned}
$$

Proof. Let $\{u(t), \omega(t)\}=\left\{u\left(t ; \tau, u_{0}, \phi_{1}\right), \omega\left(t ; \tau, \omega_{0}, \phi_{2}\right)\right\}$ be the solution of equations (3) corresponding to the initial data $\tau, u_{0}, \phi_{1}, \omega_{0}, \phi_{2}$. From

$$
\frac{\mathrm{d}}{\mathrm{d} t}|u|^{2}+v\|u\|^{2} \leq \frac{2 \xi^{2}}{v \lambda_{1}} C_{T_{2}}+\frac{2}{v \lambda_{1}}\left|G_{1}(t, u(t-\rho(t)))\right|^{2},
$$

we obtain

$$
\begin{aligned}
& \frac{\mathrm{d}}{\mathrm{d} t}\left(\mathrm{e}^{\varepsilon_{1} t}|u(t)|^{2}\right) \\
& \leq-v \mathrm{e}^{\varepsilon_{1} t}\|u(t)\|^{2}+\frac{2 \xi^{2}}{v \lambda_{1}} C_{T_{2}} \mathrm{e}^{\varepsilon_{1} t}+\frac{2}{v \lambda_{1}} \mathrm{e}^{\varepsilon_{1} t}\left|G_{1}(t, u(t-\rho(t)))\right|^{2}+\varepsilon_{1} \mathrm{e}^{\varepsilon_{1} t}|u(t)|^{2}
\end{aligned}
$$




$$
\leq\left(\varepsilon_{1}-v \lambda_{1}\right) \mathrm{e}^{\varepsilon_{1} t}|u(t)|^{2}+\frac{2 \xi^{2}}{v \lambda_{1}} C_{T_{2}} \mathrm{e}^{\varepsilon_{1} t}+\frac{2}{v \lambda_{1}} \mathrm{e}^{\varepsilon_{1} t}\left|G_{1}(t, u(t-\rho(t)))\right|^{2} .
$$

Integrating over $[\tau, t]$, we obtain

$$
\begin{aligned}
\int_{\tau}^{t} \frac{\mathrm{d}}{\mathrm{d} s}\left(\mathrm{e}^{\varepsilon_{1} s}|u(s)|^{2}\right) \mathrm{d} s \leq & \int_{\tau}^{t}\left(\left(\varepsilon_{1}-v \lambda_{1}\right) \mathrm{e}^{\varepsilon_{1} s}|u(s)|^{2}+\frac{2 \xi^{2}}{v \lambda_{1}} C_{T_{2}} \mathrm{e}^{\varepsilon_{1} s}\right. \\
& \left.+\frac{2}{v \lambda_{1}} \mathrm{e}^{\varepsilon_{1} s}\left|G_{1}(s, u(s-\rho(s)))\right|^{2}\right) \mathrm{d} s .
\end{aligned}
$$

That is

$$
\begin{aligned}
\mathrm{e}^{\varepsilon_{1} t}|u(t)|^{2} \leq & \mathrm{e}^{\varepsilon_{1} \tau}|u(\tau)|^{2}+\left(\varepsilon_{1}-v \lambda_{1}\right) \int_{\tau}^{t} \mathrm{e}^{\varepsilon_{1} s}|u(s)|^{2} \mathrm{~d} s \\
& +\frac{2 \xi^{2}}{v \lambda_{1}} \int_{\tau}^{t} C_{T_{2}} \mathrm{e}^{\varepsilon_{1} s} \mathrm{~d} s+\frac{2}{v \lambda_{1}} \int_{\tau}^{t} \mathrm{e}^{\varepsilon_{1} s}\left|G_{1}(s, u(s-\rho(s)))\right|^{2} \mathrm{~d} s .
\end{aligned}
$$

Since

$$
\begin{aligned}
& \int_{\tau}^{t} \mathrm{e}^{\varepsilon_{1} s}\left|G_{1}(s, u(s-\rho(s)))\right|^{2} \mathrm{~d} s \\
& \leq\left|g_{1}\right|_{\infty} \int_{\tau}^{t} \mathrm{e}^{\varepsilon_{1} s}|u(s-\rho(s))|^{2} \mathrm{~d} s+\int_{\tau}^{t} \mathrm{e}^{\varepsilon_{1} s} f_{1}(s) \mathrm{d} s \\
& \leq \frac{\left|g_{1}\right|_{\infty} \mathrm{e}^{\varepsilon_{1} h}}{1-\rho_{*}} \int_{\tau}^{t} \mathrm{e}^{\varepsilon_{1} s}|u(s)|^{2} \mathrm{~d} s+\frac{\left|g_{1}\right|_{\infty} \mathrm{e}^{\varepsilon_{1} h}}{1-\rho_{*}} \int_{\tau-h}^{\tau} \mathrm{e}^{\varepsilon_{1} s}\left|\phi_{1}(s-\tau)\right|^{2} \mathrm{~d} s+\int_{\tau}^{t} \mathrm{e}^{\varepsilon_{1} s} f_{1}(s) \mathrm{d} s \\
& =\frac{\left|g_{1}\right|_{\infty} \mathrm{e}^{\varepsilon_{1} h}}{1-\rho_{*}} \int_{\tau}^{t} \mathrm{e}^{\varepsilon_{\varepsilon_{1} s}}|u(s)|^{2} \mathrm{~d} s+\frac{\left|g_{1}\right|_{\infty} \mathrm{e}^{\varepsilon_{1}(h+\tau)}}{1-\rho_{*}} \int_{-h}^{0} \mathrm{e}^{\varepsilon_{1} s}\left|\phi_{1}(s)\right|^{2} \mathrm{~d} s+\int_{\tau}^{t} \mathrm{e}^{\varepsilon_{1} s} f_{1}(s) \mathrm{d} s
\end{aligned}
$$

Using (19), we can get

$$
\begin{gathered}
\mathrm{e}^{\varepsilon_{1} t}|u(t)|^{2} \\
\leq \mathrm{e}^{\varepsilon_{1} \tau}|u(\tau)|^{2}+\left(\varepsilon_{1}-v \lambda_{1}\right) \int_{\tau}^{t} \mathrm{e}^{\varepsilon_{1} s}|u(s)|^{2} \mathrm{~d} s \\
+\frac{2 \xi^{2}}{v \lambda_{1}} \int_{\tau}^{t} C_{T_{2}} \mathrm{e}^{\varepsilon_{1} s} \mathrm{~d} s+\frac{2}{v \lambda_{1}}\left(\frac{\left|g_{1}\right|_{\infty} \mathrm{e}^{\varepsilon_{1} h}}{1-\rho_{*}} \int_{\tau}^{t} \mathrm{e}^{\varepsilon_{1} s}|u(s)|^{2} \mathrm{~d} s\right. \\
\left.+\frac{\left|g_{1}\right| \mathrm{e}^{\varepsilon_{1}(h+\tau)}}{1-\rho_{*}} \int_{-h}^{0} \mathrm{e}^{\varepsilon_{1} s}\left|\phi_{1}(s)\right|^{2} \mathrm{~d} s+\int_{\tau}^{t} \mathrm{e}^{\varepsilon_{1} s} f_{1}(s) \mathrm{d} s\right) \\
=\frac{\left.2\left|g^{\varepsilon_{1} \tau}\right| u(\tau)\right|^{2}+\left(\varepsilon_{1}-v \lambda_{1}+\frac{2\left|g_{1}\right|_{\infty} \mathrm{e}^{\varepsilon_{1} h}}{v \lambda_{1}\left(1-\rho_{*}\right)}\right) \int_{\tau}^{t} \mathrm{e}^{\varepsilon_{1} s}|u(s)|^{2} \mathrm{~d} s+\frac{2 \xi^{2}}{v \lambda_{1}} \int_{\tau}^{t} C_{T_{2}} \mathrm{e}^{\varepsilon_{1} s} \mathrm{~d} s}{v \lambda_{1}\left(1-\rho_{*}\right)} \int_{-h}^{0} \mathrm{e}^{\varepsilon_{1} s}\left|\phi_{1}(s)\right|^{2} \mathrm{~d} s+\frac{2}{v \lambda_{1}} \int_{\tau}^{t} \mathrm{e}^{\varepsilon_{1} s} f_{1}(s) \mathrm{d} s \\
=\mathrm{e}^{\varepsilon_{1} \tau}|u(\tau)|^{2}+\frac{2 \xi^{2}}{v \lambda_{1}} \int_{\tau}^{t} C_{T_{2}} \mathrm{e}^{\varepsilon_{1} s} \mathrm{~d} s+\frac{2\left|g_{1}\right|_{\infty} \mathrm{e}^{\varepsilon_{1}(h+\tau)}}{v \lambda_{1}\left(1-\rho_{*}\right)} \int_{-h}^{0} \mathrm{e}^{\varepsilon_{1} s}\left|\phi_{1}(s)\right|^{2} \mathrm{~d} s \\
+\frac{2}{v \lambda_{1}} \int_{\tau}^{t} \mathrm{e}^{\varepsilon_{1} s} f_{1}(s) \mathrm{d} s .
\end{gathered}
$$

That is 
X. How, C. S. Thu

$$
\begin{aligned}
|u(t)|^{2} \leq & \mathrm{e}^{\varepsilon_{1}(\tau-t)}|u(\tau)|^{2}+\frac{2 \xi^{2}}{v \lambda_{1}} \mathrm{e}^{-\varepsilon_{1} t} \int_{\tau}^{t} C_{T_{2}} \mathrm{e}^{\varepsilon_{1} s} \mathrm{~d} s \\
& +\frac{2\left|g_{1}\right|_{\infty} \mathrm{e}^{\varepsilon_{1}(h+\tau)}}{v \lambda_{1} \mathrm{e}^{\varepsilon_{1} t}\left(1-\rho_{*}\right)} \int_{-h}^{0} \mathrm{e}^{\varepsilon_{1} s}\left|\phi_{1}(s)\right|^{2} \mathrm{~d} s+\frac{2}{v \lambda_{1}} \mathrm{e}^{-\varepsilon_{1} t} \int_{\tau}^{t} \mathrm{e}^{\varepsilon_{1} s} f_{1}(s) \mathrm{d} s \\
= & \mathrm{e}^{\varepsilon_{1}(\tau-t)}\left(|u(\tau)|^{2}+\frac{2\left|g_{1}\right|_{\infty} \mathrm{e}^{\varepsilon_{1} h}}{v \lambda_{1}\left(1-\rho_{*}\right)} \int_{-h}^{0} \mathrm{e}^{\varepsilon_{1} s}\left|\phi_{1}(s)\right|^{2} \mathrm{~d} s\right) \\
& +\mathrm{e}^{-\varepsilon_{1} t}\left(\frac{2}{v \lambda_{1}} \int_{\tau}^{t} \mathrm{e}^{\varepsilon_{1} s} f_{1}(s) \mathrm{d} s+\frac{2 \xi^{2}}{v \lambda_{1}} \int_{\tau}^{t} \mathrm{e}^{\varepsilon_{1} s} C_{T_{2}} \mathrm{~d} s\right) .
\end{aligned}
$$

Similarly, from

$$
\frac{\mathrm{d}}{\mathrm{d} t}|\omega|^{2}+v\|\omega\|^{2} \leq \frac{1}{\lambda_{1}}\left|G_{2}(t, \omega(t-\rho(t)))\right|^{2},
$$

we obtain

$$
\begin{aligned}
& \frac{\mathrm{d}}{\mathrm{d} t}\left(\mathrm{e}^{\varepsilon_{2} t}|\omega(t)|^{2}\right) \\
& \leq-\left.\mathrm{e}^{\varepsilon_{2} t}\left|\omega(t) \|^{2}+\varepsilon_{2} \mathrm{e}^{\varepsilon_{2} t}\right| \omega(t)\right|^{2}+\frac{1}{\lambda_{1}} \mathrm{e}^{\varepsilon_{2} t}\left|G_{2}(t, \omega(t-\rho(t)))\right|^{2} \\
& \leq\left(\varepsilon_{2}-\lambda_{1}\right) \mathrm{e}^{\varepsilon_{2} t}|\omega(t)|^{2}+\frac{1}{\lambda_{1}} \mathrm{e}^{\varepsilon_{2} t}\left|G_{2}(t, \omega(t-\rho(t)))\right|^{2} .
\end{aligned}
$$

Integrating over $[\tau, t]$, we obtain

$$
\begin{aligned}
& \int_{\tau}^{t} \frac{\mathrm{d}}{\mathrm{d} s}\left(\mathrm{e}^{\varepsilon_{2} s}|\omega(s)|^{2}\right) \mathrm{d} s \\
& \leq \int_{\tau}^{t}\left(\left(\varepsilon_{2}-\lambda_{1}\right) \mathrm{e}^{\varepsilon_{2} s}|\omega(s)|^{2}+\frac{1}{\lambda_{1}} \mathrm{e}^{\varepsilon_{2} s}\left|G_{2}(s, \omega(s-\rho(s)))\right|^{2}\right) \mathrm{d} s .
\end{aligned}
$$

Using (20) and (24), we can get

$$
\begin{aligned}
& \mathrm{e}^{\varepsilon_{2} t}|\omega(t)|^{2} \\
& \leq \mathrm{e}^{\varepsilon_{2} \tau}|\omega(\tau)|^{2}+\left(\varepsilon_{2}-\lambda_{1}\right) \int_{\tau}^{t} \mathrm{e}^{\varepsilon_{2} s}|\omega(s)|^{2} \mathrm{~d} s+\frac{1}{\lambda_{1}} \int_{\tau}^{t} \mathrm{e}^{\varepsilon_{2} s}\left|G_{2}(s, \omega(s-\rho(s)))\right|^{2} \mathrm{~d} s \\
& \leq \mathrm{e}^{\varepsilon_{2} \tau}|\omega(\tau)|^{2}+\left(\varepsilon_{2}-\lambda_{1}\right) \int_{\tau}^{t} \mathrm{e}^{\varepsilon_{2} s}|\omega(s)|^{2} \mathrm{~d} s+\frac{1}{\lambda_{1}}\left(\frac{\left|g_{2}\right|_{\infty} \mathrm{e}^{\varepsilon_{2} h}}{1-\rho_{*}} \int_{\tau}^{t} \mathrm{e}^{\varepsilon_{2} s}|\omega(s)|^{2} \mathrm{~d} s\right. \\
& \left.+\frac{\left|g_{2}\right|_{\infty} \mathrm{e}^{\varepsilon_{2}(h+\tau)}}{1-\rho_{*}} \int_{-h}^{0} \mathrm{e}^{\varepsilon_{2} s}\left|\phi_{2}(s)\right|^{2} \mathrm{~d} s+\int_{\tau}^{t} e^{\varepsilon_{2} s} f_{2}(s) \mathrm{d} s\right) \\
& =\mathrm{e}^{\varepsilon_{2} \tau}|\omega(\tau)|^{2}+\left(\varepsilon_{2}-\lambda_{1}+\frac{\left|g_{2}\right|_{\infty} \mathrm{e}^{\varepsilon_{2} h}}{\lambda_{1}\left(1-\rho_{*}\right)}\right) \int_{\tau}^{t} \mathrm{e}^{\varepsilon_{2} s}|\omega(s)|^{2} \mathrm{~d} s \\
& \quad+\frac{\left|g_{2}\right|_{\infty} \mathrm{e}^{\varepsilon_{2}(h+\tau)}}{\lambda_{1}\left(1-\rho_{*}\right)} \int_{-h}^{0} \mathrm{e}^{\varepsilon_{2} s}\left|\phi_{2}(s)\right|^{2} \mathrm{~d} s+\frac{1}{\lambda_{1}} \int_{\tau}^{t} \mathrm{e}^{\varepsilon_{2} s} f_{2}(s) \mathrm{d} s \\
& =\mathrm{e}^{\varepsilon_{2} \tau}|\omega(\tau)|^{2}+\frac{\left|g_{2}\right|_{\infty} \mathrm{e}^{\varepsilon_{2}(h+\tau)}}{\lambda_{1}\left(1-\rho_{*}\right)} \int_{-h}^{0} \mathrm{e}^{\varepsilon_{2} s}\left|\phi_{2}(s)\right|^{2} \mathrm{~d} s+\frac{1}{\lambda_{1}} \int_{\tau}^{t} \mathrm{e}^{\varepsilon_{2} s} f_{2}(s) \mathrm{d} s .
\end{aligned}
$$

That is

DOI: 10.4236/oalib.1105163

13

Open Access Library Journal 


$$
\begin{aligned}
& |\omega(t)|^{2} \\
& \leq \mathrm{e}^{\varepsilon_{2}(\tau-t)}|\omega(\tau)|^{2}+\frac{\left|g_{2}\right|_{\infty} \mathrm{e}^{\varepsilon_{2}(h+\tau)}}{\lambda_{1}\left(1-\rho_{*}\right)} \mathrm{e}^{-\varepsilon_{2} t} \int_{-h}^{0} \mathrm{e}^{\varepsilon_{2} s}\left|\phi_{2}(s)\right|^{2} \mathrm{~d} s+\frac{1}{\lambda_{1}} \mathrm{e}^{-\varepsilon_{2} t} \int_{\tau}^{t} \mathrm{e}^{\varepsilon_{2} s} f_{2}(s) \mathrm{d} s \\
& =\mathrm{e}^{\varepsilon_{2}(\tau-t)}\left(|\omega(\tau)|^{2}+\frac{\left|g_{2}\right|_{\infty} \mathrm{e}^{\varepsilon_{2} h}}{\lambda_{1}\left(1-\rho_{*}\right)} \int_{-h}^{0} \mathrm{e}^{\varepsilon_{2} s}\left|\phi_{2}(s)\right|^{2} \mathrm{~d} s\right)+\frac{1}{\lambda_{1}} \mathrm{e}^{-\varepsilon_{2} t} \int_{\tau}^{t} \mathrm{e}^{\varepsilon_{2} s} f_{2}(s) \mathrm{d} s .
\end{aligned}
$$

\section{Conflicts of Interest}

The authors declare no conflicts of interest regarding the publication of this paper.

\section{References}

[1] Temam, R. (1988) Infinite-Dimensional Dynamical Systems in Mechanics and Physics. Springer-Verlag, New York. https://doi.org/10.1007/978-1-4684-0313-8

[2] Birnir, B. and Svanstedt, N. (2004) Existence Theory and Strong Attractors for the Rayleigh-Bénard Problem with a Large Aspect Ratio. Discrete \& Continuous Dynamical Systems, 10, 53-74.

[3] Caraballo, T., Márquez-Durán, A.M. and Real, J. (2010) Three-Dimensional System of Globally Modified Navier-Stokes Equations with Delay. International Journal of Bifurcation and Chaos in Applied Sciences, 20, 2869-2883. https://doi.org/10.1142/S0218127410027428

[4] Marn-Rubio, P., Márquez-Durán, A.M. and Real, J. (2013) Asymptotic Behavior of Solutions for a Three Dimensional System of Globally Modified Navier-Stokes Equations with a Locally Lipschitz Delay Term. Nonlinear Analysis, 79, 68-79. https://doi.org/10.1016/j.na.2012.11.006

[5] Romito, M. (2009) The Uniqueness of Weak Solutions of the Globally Modified Navier-Stokes Equations. Advanced Nonlinear Studies, 9, 425-429. https://doi.org/10.1515/ans-2009-0209

[6] Robinson, J.C. (2001) Infinite-Dimensional Dynamical Systems. Cambridge University Press, Cambridge. https://doi.org/10.1007/978-94-010-0732-0

[7] Kapustyan, O.V., Melnik, V.S. and Valero, J. (2007) A Weak Attractor and Properties of Solutions for the Three-Dimensional Bénard Problem. Discrete \& Continuous Dynamical Systems, 18, 449-481. https://doi.org/10.3934/dcds.2007.18.449

[8] Temam, R. (1979) Navier-Stokes Equations. North-Holland, Amsterdam.

[9] Lions, J.L. (1969) Quelques Méthodes de Résolution des Problèmes aux Limites Non Linéaires. Dunod, Paris.

[10] Evans, L.C. (2010) Partial Differential Equations. American Mathematical Society, 22, 261-271. https://doi.org/10.1090/gsm/019 